\title{
Weyl type upper bounds on the number of resonances near the real axis for trapped systems
}

\author{
Plamen Stefanov
}

\begin{abstract}
We study semiclassical resonances in a box $\Omega(h)$ of height $h^{N}, N \gg 1$. We show that the semiclassical wave front set of the resonant states (including the "generalized eigenfunctions") is contained in the set $\mathcal{T}$ of the trapped bicharacteristics. We also show that for a suitable self-adjoint reference operator $P^{\#}(h)$ with discrete spectrum the number of resonances in $\Omega(h)$ is bounded by the number of eigenvalues of $P^{\#}(h)$ in an interval a bit larger than the projection of $\Omega(h)$ on the real line. As an application, we prove a Weyl type estimate of the number of resonances in $\Omega(h)$ in terms of the measure of $\mathcal{T}$. We prove a similar estimate in case of classical scattering by a metric and obstacle.
\end{abstract}

\section{Introduction}

We study resonances in semiclassical scattering in a box $\Omega(h)=\left[a_{0}, b_{0}\right]+i[-S(h), 0]$, where $0<S(h)=O\left(h^{K}\right), K \gg 1$. In the classical case, we study resonances $\lambda$ with $\operatorname{Re} \lambda \gg 1,0 \leq-\operatorname{Im} \lambda \leq C(\operatorname{Re} \lambda)^{-K}, K \gg 1$. Such resonances may exist only for trapping geometries if the trapping is "strong" enough. Here we will formulate the main results and explain the main ideas, the full proofs will be published elsewhere.

We work in the abstract "black box scattering" setting in $\mathbf{R}^{n}$, introduced by Sjöstrand and Zworski [SjZ] and developed further by Sjöstrand [Sj2] for long range operators. Let $P(h)$ be such a semiclassical long range operator. First we study the properties of the resonant states related to resonances near the real axis. Resonant states are all outgoing "eigenfunctions" $u(h)$ (of infinite energy) that solve the equation $(P(h)-z(h)) u(h)=0$ and "generalized eigenfunctions" belonging to the range of the residue of the resolvent at the resonance $z(h)$ that solve $(P(h)-z(h))^{m} u(h)=0$ with some $m$. In view of the a priori polynomial bound $N(\Omega(h))=O\left(h^{-n^{\#}}\right)$, $n^{\#} \geq n$, where $N(\Omega(h))$ is the number of resonances in $\Omega(h)$, counting multiplicities, we have that $m=O\left(h^{-n^{\#}}\right)$. We prove that all resonant states satisfy

Supported by NSF Grant DMS-0070823

$M S C$ 2000: 35P25.

Keywords : resonances, trapping. 
$(P(h)-z(h)) u(h)=O\left(h^{K_{1}}\right)$, where $K_{1}$ depends on $K$. We also prove that for some $K_{2}=K_{2}(K) \gg 1$, all normalized resonant states are $O\left(h^{K_{2}}\right)$ small, in an annulus $R_{1} \leq|x| \leq R_{2}$ surrounding the black box and this estimate is uniform with respect to the resonances and the order $m$ of the resonant state, as long as $z(h) \in \Omega(h)$. In fact, we do not work with single resonances but with clusters of resonances and prove the above estimates for any normalized linear combination of resonant states belonging to such a cluster. Next, we show that the linear independence of the resonant states belonging to different clusters in $\Omega(h)$ is stable under certain small perturbations. If we work with eigenvalues of a self-adjoint operator, this is true because of the orthogonality of the eigenfunctions, in the case under consideration this stability replaces the orthogonality property. Those results allow us to show that all resonances in $\Omega(h)$ are perturbations of some of the eigenvalues of a suitable "reference" self-adjoint operator with discrete spectrum.

In the case when $P(h)$ is a second order elliptic semiclassical PDO, we use propagation of singularities arguments to show that the resonant states are "small" not only far away from the scatterer, but also they are "small" microlocally along all non-trapped bicharacteristics. In other words, for a suitable $s=s(K)$, with $s \gg 1$, if $K \gg 1$, we show that the semiclassical wave front set $\mathrm{WF}^{s}(u)$ is included in the trapped set $\mathcal{T} \subset T^{*} \mathbf{R}$ defined as the set of those $(x, \xi)$ for which the bicharacteristic through $(x, \xi)$ never leaves some compact set. This allows us to construct a reference operator $P^{\#}(h)$ from $P(h)$ by adding a barrier near the trapped set and comparing the spectral density of $P^{\#}(h)$ and $N(\Omega(h))$ as above, to get Weyl type upper bounds on $N(\Omega(h)$ ) in terms of the measure of $\mathcal{T}$. We get a similar estimate in the classical case of scattering by obstacle (and metric).

The idea that the counting function of the resonances (not only near the real line) is essentially bounded by the spectral counting function of certain reference operator, which is $P(h)$, modified for $|x|>R_{0}$, has been used implicitly or explicitely [Z1], [SjZ], [V], [Sj2] in the proof of the polynomial bound of this function. Sjöstrand [Sj1], under certain assumptions involving analyticity and hyperbolicity of the bicharacteristic flow, showed that the resonances in a box of height $\delta, C_{0} h \leq \delta \leq 1 / C_{0}$ is $O\left(\delta^{d-\varepsilon} h^{-n}\right), \varepsilon>0$, where $d$ is the Minkowski codimension of the set of the trapped rays. Numerical study of this and other phenomena can be found in [LZ]. M. Zerzeri [Ze] obtained in the classical case an upper bound in a sector in $\mathbf{C}$ related to the measure of the trapped rays but the notion of trapped rays that he uses is weaker than the common one.

\section{Estimates on the resonant states}

In the black-box scattering [SjZ], [Sj2] one works with self-adjoint operator $P(h)$ on the Hilbert space $\mathcal{H}=\mathcal{H}_{R_{0}} \oplus L^{2}\left(\mathbf{R}^{n} \backslash B\left(0, R_{0}\right)\right)$. We refer to [Sj2] for more details. Important examples are the long range Schrödinger operator $-h^{2} \Delta+V(x)$ with $V(x)$ analytic in $x$ in the domain $\{r \omega ; \omega \in \mathbf{C}\}, r>R_{0}, \operatorname{dist}\left(\omega, S^{n-1}\right)<$ $\left.d_{0}, r \in \mathbf{C}, \arg (r) \in\left[-\theta_{0}, \theta_{0}\right]\right\}$, or the classical or semiclassical Laplacian outside a bounded obstacle with Dirichlet or Neumann boundary conditions. For simplicity we assume below that in $\mathbf{R}^{n} \backslash B\left(0, R_{0}\right)$ we have $P(h)=-h^{2} \Delta+V(x)$, where $V(x)$ is a long range potential as above but our results remain true for more general long 
range perturbations of the semiclassical Laplacian as in [Sj2]. The (semi-classical) resonances $\operatorname{Res} P(h)$ of $P(h)$ are defined in a conic neighborhood of the real axis by the method of complex scaling (see [SjZ], [Sj2]).

As in [SjZ], [Sj2], we construct a reference selfadjoint operator $P^{\#}(h)$ from $P(h)$ on $\mathcal{H}^{\#}=\mathcal{H}_{R_{0}} \oplus L^{2}\left(M \backslash B\left(0, R_{0}\right)\right)$, where $M=(\mathbf{R} / R \mathbf{Z})^{n}$ for some $R \gg R_{0}$. Then for the number of eigenvalues of $P^{\#}$ in a given interval $[-\lambda, \lambda]$, we assume

$$
\#\left\{z \in \operatorname{Spec} P^{\#}(h) ;-\lambda \leq z \leq \lambda ;\right\} \leq C\left(\lambda / h^{2}\right)^{n^{\#} / 2}, \quad \lambda \geq 1,
$$

with some $n^{\#} \geq n$. This implies (see [SjZ] and [Sj2]) that

$$
\#\left\{z \in \operatorname{Res} P(h) ; 0<a_{0} \leq \operatorname{Re} z \leq b_{0} ; 0 \leq-\operatorname{Im} z \leq c_{0}\right\} \leq C\left(a_{0}, b_{0}, c_{0}\right) h^{-n^{\#}} .
$$

For any resonance $z(h)$ there is an outgoing solution $u(h)$ to $(P(h)-z(h)) u(h)=$ 0 and possibly "generalized eigenvectors" $v(h)$ satisfying $(P(h)-z(h))^{k(h)} v(h)=0$. We will call $u$ and $v$ resonant states. Given $\Omega(h) \subset \mathbf{C}, N(\Omega(h))$ will denote the number of resonances in $\Omega(h)$ counted with their multiplicities defined as the rank of the residue of the cut-off resolvent at any resonance.

For technical reasons, we work with the complex scaled operator $P_{\theta}(h)$. Fix $A<B$, such that $R_{0}+1<A<B-1$. As in [SjZ] and [Sj2], we choose a real-valued increasing $C^{\infty}$-function $\kappa(r), r \geq 0$, with the properties:

(i) $\kappa(r)=0$ for $0 \leq r \leq B$,

(ii) $\kappa(r)=1$ for $r \geq B+1 / 2$,

(iii) $0 \leq \kappa(r) \leq 1$,

(iv) $\kappa(r)=e^{-1 /(r-B)^{2}}$ for $B \leq r \leq B+\epsilon_{0}$ with some $\epsilon_{0} \ll 1$.

Set $\theta=\theta(r):=\theta_{0} \kappa(r)$, where $0<\theta_{0} \ll 1$ will be chosen later. Define $f_{\theta}(r):=r e^{i \theta(r)}$. As in [SjZ], [Sj2], we perform the analytic dilation by considering the map

$$
\mathbf{R}^{n} \ni x=r \omega \longmapsto f_{\theta}(r) \omega \in \mathbf{C}^{n}, \quad \omega \in S^{n-1} .
$$

This transforms $P$ into an operator $P_{\theta}$ on $\mathcal{H}_{R_{0}} \oplus L^{2}\left(\Gamma_{\theta} \backslash B\left(0, R_{0}\right)\right)$, where $\Gamma_{\theta}$ is the image of (2). We always identify $\Gamma_{\theta}$ with $\mathbf{R}^{n}$.

It is known that for a fixed $h>0$ and $z \neq 0$ with $\arg (z) \neq-2 \theta_{0}$, the operator $P_{\theta}-z$ is Fredholm with index 0 . Moreover, for $z \neq 0$ with $-\arg (z)<2 \theta_{0}$, and in particular for $z \in \Omega(h)$, we have that $z$ is a resonance of $P$ if and only if $z$ is an eigenvalue of $P_{\theta}$ and the multiplicities coincide.

Our first starting point is an estimate that is a refinement of [B2, Prop. 6.1]. Let $\rho(r)$ be a smooth function equal to 1 for $r<A-1 / 2$ and equal to $r^{(n-1) / 2}$ for $r>A$. Set $\tilde{P}:=\rho P \rho^{-1}$. Then $\tilde{P}$ is self-adjoint for the measure $d \mu:=\rho^{-2} r^{n-1} d r d \omega$ and we denote by $\tilde{\mathcal{H}}=\rho \mathcal{H}$ the corresponding Hilbert space.

Let $\tilde{P}_{\theta}$ be the operator obtained from $\tilde{P}$ by analytic dilation for $r \geq B$ and denote by $\tilde{\mathcal{D}}$ its domain. 
Proposition 1 There exists $B_{0}>0$, such that if $B \geq B_{0}$, then for $h>0$ and $\theta_{0}>0$ small enough, $\operatorname{Re} z \geq a_{0}, \operatorname{Im} z \leq 0$, and for any $u \in \tilde{D}_{\theta}$ we have

$$
\begin{aligned}
C \int\left(\left(\theta+r \theta^{\prime}\right)\left|h \partial_{r} u\right|^{2}\right. & \left.+\theta\left(\left|h r^{-1} \nabla_{\omega} u\right|^{2}+|u|^{2}\right)\right) d r d \omega \\
\leq & -\operatorname{Im}\left(e^{i \theta}\left(\tilde{P}_{\theta}-z\right) u, u\right)_{\tilde{\mathcal{H}}}+\left(-\operatorname{Im} z+e^{-h^{-1 / 3}}\right)\|u\|_{\tilde{\mathcal{H}}}^{2},
\end{aligned}
$$

where $C=\min \left(a_{0}, 1\right) / 2$ and the inner product and the norm are taken in $\tilde{\mathcal{H}}$.

For a proof, we refer to [B2]. The only new point here is that we give a different form of the error term represented here by $e^{-h^{-1 / 3}}$.

We note that the estimate above remains true for $\operatorname{Im} z>0$ as well after replacing $-\operatorname{Im} z$ by $-\cos \theta_{0} \operatorname{Im} z$. This shows that

$$
\left\|\left(P_{\theta}-z\right)^{-1}\right\| \leq \frac{2}{\operatorname{Im} z-e^{-h^{-1 / 3}}}, \quad \operatorname{Im} z>e^{-h^{-1 / 3}} .
$$

The second starting point is an estimate of the resolvent on a contour around a cluster of resonances. Fix $0<a_{0}<b_{0}$. Choose some $a(h), b(h)$ and $c(h)$ such that

$$
a_{0} \leq a(h) \leq b(h) \leq b_{0}, \quad b(h)-a(h)=o(1), \quad 2 e^{-h^{-1 / 3}} \leq c(h) \leq o(1) h^{\left(5 n^{\#}+1\right) / 2}
$$

and let

$$
\Omega(h):=[a(h), b(h)]+i[-c(h), 0] .
$$

Let $z_{1}(h), \ldots, z_{p}(h)$ be all distinct resonances in $\Omega(h)$ with multiplicities $m_{1}(h), \ldots$, $m_{p}(h)$. Set $m(h):=m_{1}+\cdots+m_{p}=N(\Omega(h))$. Assume that there are no resonances on $\partial \Omega$. Consider the spectral projector associated with the eigenvalues of $P_{\theta}$ in $\Omega$

$$
\Pi_{\Omega}:=\frac{1}{2 \pi i} \oint_{\partial \Omega}\left(z-P_{\theta}\right)^{-1} d z
$$

where $\partial \Omega$ is assumed to be positively oriented. Denote $\mathcal{H}_{\Omega}:=\operatorname{Ran} \Pi_{\Omega}$. Then it is well known (see e.g. $[\mathrm{K}]$ ) that $P_{\theta}$ acts invariantly on $\mathcal{H}_{\Omega}$ that is the span of all eigenvectors and generalized eigenvectors of $P_{\theta}$ with eigenvalues in $\Omega$. The dimension $m(h)$ of $\mathcal{H}_{\Omega}$ is finite, bounded by $C h^{-n \#}$, and equal to the sum of the multiplicities of $z_{j} \in \Omega$. Set $P_{\Omega}:=\left.P_{\theta}\right|_{\mathcal{H}_{\Omega}}$. Then $P_{\Omega}$ is a finite rank operator (matrix) and we denote by $\|\cdot\|_{\mathcal{H}_{\Omega}}$ the operator norm in $\mathcal{H}_{\Omega}$. The spectrum of $P_{\Omega}$ consists of $\left\{z_{1}, \ldots, z_{p}\right\}$ with the same multiplicities. We need the following estimate due to Zworski [Z2] (in this generality, see the proof of Lemma 1 in [TZ1])

$\left\|\left(z-P_{\theta}\right)^{-1}\right\| \leq C e^{C h^{-n \#} \ln (1 / g)} \quad$ for $z \in \Omega_{0}, \operatorname{dist}\left(z, \operatorname{Res} P(h) \cap \Omega_{0}\right) \geq g(h), g(h) \ll 1$,

where $\Omega_{0}$ is any simply connected precompact subset of $-\pi<-\arg z<2 \theta_{0}$ (independent of $h$ ). In our analysis $\Omega(h)$ is always included in such a fixed set, therefore the constant $C$ above will be uniform. As a consequence, the resolvent of $P_{\Omega}$ satisfies the following estimate

$$
\left\|\left(z-P_{\Omega}\right)^{-1}\right\|_{\mathcal{H}_{\Omega}} \leq C e^{C h^{-n^{\#}} \ln (1 / g)} \quad \text { for } z \in \Omega_{0}, \operatorname{dist}\left(z, \operatorname{Res} P(h) \cap \Omega_{0}\right) \geq g(h),
$$

where $g(h) \ll 1$. This allows us to apply the "semiclassical maximum principle" ([TZ1], [TZ2]) as in [St3, Lemma 2] to get the following. 
Proposition 2 Assume that $c(h) \leq S(h) \leq h^{\left(5 n^{\#}+1\right) / 2} w(h), w(h)=o(1)$, as $h \rightarrow 0$, where $a(h), b(h)$ and $c(h)$ are as in (5). Then

$$
\left\|\left(z-P_{\Omega}\right)^{-1}\right\|_{\mathcal{H}_{\Omega}} \leq \frac{C}{S(h)} \quad \text { on } \partial \tilde{\Omega}
$$

where $\tilde{\Omega}:=[a(h)-w(h), b(h)+w(h)]+\left[-h^{-n^{\#}} S(h), S(h)\right]$.

Sketch of the proof. The proof is similar to that of [St3, Lemma 2]. We multiply $\left(z-P_{\Omega}\right)^{-1}$ by

$$
G(z, h):=\frac{\left(z-z_{1}\right)^{m_{1}} \ldots\left(z-z_{p}\right)^{m_{p}}}{\left(z-\tilde{z}_{1}\right)^{m_{1}} \ldots\left(z-\tilde{z}_{p}\right)^{m_{p}}}
$$

where $\tilde{z}_{j}(h):=\bar{z}_{j}(h)+2 i S(h), j=1 \ldots p$, are obtained from $z_{j}(h)$ by reflection about the line $\operatorname{Im} z=S(h)$. On that line we have $\left\|\left(z-P_{\Omega}\right)^{-1}\right\|_{\mathcal{H}_{\Omega}} \leq 4 / S(h)$ by (5) and (4). We have $|G(z, h)| \leq 1$ for $\operatorname{Im} z \leq S(h)$. The function $F:=G\left(z-P_{\Omega}\right)^{-1}$ is holomorphic below the line $\operatorname{Im} z=S(h)$, and in particular in $\Omega(h)$. This allows us to apply the "semiclassical maximum principle" [TZ1] in the form presented in [St3, Lemma 1] to the function $F$ in the domain $\Omega_{1}:=[a(h)-5 w(h), b(h)+5 w(h)]+$ $i\left[-S(h) h^{-2 n^{\#}-1}, S(h)\right]$ to get that for $h$ small enough

$$
\left\|G(z)\left(z-P_{\Omega}\right)^{-1}\right\|_{\mathcal{H}_{\Omega}} \leq 2 e^{3} / S(h), \quad \forall z \in \tilde{\Omega}(h) .
$$

Next we show that $1 / C \leq|G(z, h)|$ on $\partial \tilde{\Omega}(h)$. This shows that (8) remains true with a different constant if we remove the factor $G(z)$ there and this completes the proof of the proposition.

This proposition allows us to estimate $\left\|P_{\Omega}-z_{0}\right\|_{\mathcal{H}_{\Omega}}$ for $z_{0} \in[a(h), b(h)]$. We have

$$
z_{0}-P_{\Omega}=\frac{1}{2 \pi i} \oint_{\partial \tilde{\Omega}}\left(z_{0}-P_{\Omega}\right)\left(z-P_{\Omega}\right)^{-1} d z=\frac{1}{2 \pi i} \oint_{\partial \tilde{\Omega}}\left(z_{0}-z\right)\left(z-P_{\Omega}\right)^{-1} d z,
$$

therefore,

$$
\left\|z_{0}-P_{\Omega}\right\|_{\mathcal{H}_{\Omega}} \leq \frac{|\partial \tilde{\Omega}|}{2 \pi}\left|z_{0}-z\right|\left\|\left(z-P_{\Omega}\right)^{-1}\right\|_{\mathcal{H}_{\Omega}} \leq C \frac{(b-a+w)^{2}}{S}
$$

Choosing $w(h)=h^{-\left(5 n^{\#}+1\right) / 2} S(h)$, and minimizing with respect to $S(z)$, we see that estimate (9) implies the following.

Proposition 3 Let $\Omega$ and $\Pi_{\Omega}$ be as above. Then for $z_{0} \in[a(h), b(h)]$ we have

$$
\left\|\left(P_{\theta}-z_{0}\right) f\right\| \leq C h^{-\left(5 n^{\#}+1\right) / 2} \max \{b(h)-a(h), c(h)\}\|f\|, \quad \forall f \in \operatorname{Ran}_{\Omega} .
$$

In particular, if $f$ is a resonant state, corresponding to a single resonance $z_{0}(h)$, then

$$
\left\|\left(P_{\theta}(h)-z_{0}(h)\right) f\right\| \leq C h^{-\left(5 n^{\#}+1\right) / 2} \max \left\{-\operatorname{Im} z_{0}(h), e^{-h^{-1 / 3}}\right\}\|f\| .
$$

The estimate in Proposition 3 above is interesting only if the width of $\Omega(h)$ does not exceed $h^{N}, N \gg\left(5 n^{\#}+1\right) / 2$. We apply this estimate to domains containing clusters of resonances as follows. Let

$$
a_{0} \leq a(h)<b(h) \leq b_{0}, \quad 2 e^{-h^{-1 / 3}} \leq c(h) \leq o(1) h^{\left(5 n^{\#}+1\right) / 2}
$$


and let $\Omega(h)$ be as in (6). Assume that there are no resonances on $\partial \Omega(h)$. A direct consequence of (1) is that one can group the resonances in $\Omega(h)$ into clusters contained in the interiors of the boxes

$$
\Omega_{k}(h)=\left[a_{k}(h), b_{k}(h)\right]+i[-c(h), 0], \quad k=1, \ldots, K(h), K=O\left(h^{-n^{\#}}\right),
$$

where $\Omega_{k}(h)$ do not intersect, moreover, $\operatorname{dist}\left\{\Omega_{k}, \Omega_{m}\right\} \geq 4 w(h)$ for $k \neq m$ and $\operatorname{width}\left(\Omega_{k}\right)=b_{k}-a_{k} \leq C h^{-n^{\#}} w(h)$, where $0<w(h)=o(1) h^{n^{\#}}$ is fixed in advance. There are no resonances in $\Omega$ outside $\Omega_{k}$ 's. Denote as before by $\Pi_{\Omega_{k}}$ the spectral projectors related to the eigenvalues of $P_{\theta}$ in $\Omega_{k}$ and let $P_{\Omega}, \mathcal{H}_{\Omega}$ be as before.

We know that the subspaces $\operatorname{Ran} \Omega_{k}(h)$ are linearly independent. The following proposition basically gives us control over the lower bound of the angles between them.

Proposition 4 Under the assumptions above, if $w(h)=h^{-\left(5 n^{\#}+1\right) / 2} c(h)$, then there exists a constant $A=A\left(a_{0}, b_{0}\right)$, such that

$$
\left\|\Pi_{\Omega_{k}}\right\|_{\mathcal{H}_{\Omega}} \leq A h^{-\left(7 n^{\#}+1\right) / 2}, \quad k=1, \ldots, K
$$

For any $f_{k} \in \operatorname{Ran} \Omega_{k}(h), k=1, \ldots, K$, and for any $k_{0}$ we have

$$
\left\|f_{k_{0}}\right\| \leq A h^{-\left(7 n^{\#}+1\right) / 2}\left\|f_{1}+\cdots+f_{K}\right\|
$$

Sketch of the proof. As in Proposition 2, we get that

$$
\left\|\left(z-P_{\Omega}\right)^{-1}\right\|_{\mathcal{H}_{\Omega}} \leq \frac{C}{c(h)} \quad \text { on } \partial \tilde{\Omega}_{k}(h), \forall k
$$

where $\tilde{\Omega}_{k}(h):=\left[a_{k}(h)-w(h), b_{k}(h)+w(h)\right]+i\left[-h^{-n^{\#}} c(h), c(h)\right]$. Note that $\tilde{\Omega}_{k}(h)$ have the same properties as $\Omega_{k}(h)$ concerning the distance between two such domains and their widths, with $w(h)$ replaced by $w(h) / 2$. To justify $(14)$, it is enough to note that in the proof of Proposition 2 we used the fact that there are no poles of $\left(z-P_{\Omega}\right)^{-1}$ above $\operatorname{Im} z=c(h)$ only, and the fact that there might be poles to the left or right of $\Omega_{k}$ does not play any role as far as those poles are separated by distance $2 w(h)$ (see also [St3]). Notice also that the constant $C$ in (14) is independent of $k$. Since there are no eigenvalues of $P_{\Omega}$ in $\tilde{\Omega}_{k} \backslash \Omega_{k}$, one can define $\left.\Pi_{\Omega_{k}}\right|_{\mathcal{H}_{\Omega}}$ as integrals of $\left(z-P_{\Omega}\right)^{-1}$ over $\partial \tilde{\Omega}_{k}$. A direct estimation of that integral, using (14), yields the proof of the first part.

To prove the second part, write

$$
f_{k_{0}}=\Pi_{\Omega_{k_{0}}}\left(f_{1}+\cdots+f_{K}\right)
$$

and use the estimate on $\Pi_{\Omega_{k_{0}}}$.

The estimates proven above allow us to estimate the number $N(\Omega(h))$ of resonances in $\Omega(h)$ from above by the number of eigenvalues of a suitably chosen reference self-adjoint operator $P^{\#}(h)$. To this end we will show first that by cutting off the resonant states outside a large ball, we get quasimodes that are also quasimodes for any such reference operator. Note that next theorem is in some sense 
converse to [St1, Theorem 1] that states that locally existence of quasimodes implies existence of resonances nearby.

Define the smooth cut-off function $0 \leq \chi_{B}(x) \leq 1$ as follows:

$$
\chi_{B}(x)=1 \text { for }|x| \leq B+3 / 4, \quad \chi_{B}(x)=0 \text { for }|x|>B+1 .
$$

In what follows, $H^{s}$ is equipped with the semiclassical norm.

Theorem 1 Let $a_{0} \leq a(h)<b(h) \leq b_{0}, b(h)-a(h)=o(1) h^{\left(5 n^{\#}+1\right) / 2}, 2 e^{-h^{-1 / 3}} \leq$ $c(h)=o(1) h^{\left(5 n^{\#}+1\right) / 2}$ and set

$$
\Omega(h)=[a(h), b(h)]+i[-c(h), 0] .
$$

Suppose that $P(h)$ has $m(h)=N(\Omega(h))$ resonances (counting multiplicities) in $\Omega(h)$. Fix $z_{0}(h) \in[a(h), b(h)]$. Then $z_{0}$ is a quasimode of multiplicity $m(h)$ for $P(h)$, in the following sense: The space $\chi_{B} \operatorname{Ran} \Pi_{\Omega}$ has dimension $m(h)$ and for any $\psi \in \chi_{B} \operatorname{Ran} \Pi_{\Omega}$ with $\|\psi\|=1$ we have

(a) $\operatorname{supp} \psi \subset B(0, B+1)$,

(b) $\left\|\left(P(h)-z_{0}(h)\right) \psi\right\| \leq C \epsilon(h)$,

where $\epsilon(h)=h^{-\left(5 n^{\#}+1\right) / 4}\{\max \{b(h)-a(h), c(h)\}\}^{1 / 2}$.

Moreover, if $\psi=\chi_{B} f$ with $f \in \chi_{B} \operatorname{Ran} \Pi_{\Omega}$, and $\|\psi\|=1$, then

$$
\|\psi-f\|_{H^{1}} \leq C \epsilon(h)
$$

Remark. It follows from the propagation of singularities arguments later and from the theorem above, that one can cut off $f$ for $B_{0}<|x|<B$, before the complex scaling is performed, if $\epsilon(h)=O\left(h^{N}\right), N>\left(5 n^{\#}+1\right) / 2$ or $N=\infty$. In other words, one can replace $f$ above by non-scaled resonant states.

Sketch of the proof of Theorem 1. Given $f \in \operatorname{Ran} \Pi_{\Omega}$, set $\psi=\chi_{B} f$. Then $\psi$ is supported in $B(0, B+1)$. First, observe that by Proposition 1 and Proposition 3,

$$
\int \theta\left(|h \nabla f|^{2}+|f|^{2}\right) d x \leq C\left(\epsilon^{2}(h)+e^{-h^{-1 / 3}}\right)\|f\|^{2} \leq C \epsilon^{2}(h)\|f\|^{2}, \quad \forall f \in \operatorname{Ran} \Pi_{\Omega}
$$

with $C>0$ independent of $f$ (and $h$ ). Since for $|x|>B+1 / 2$ we have $\theta=\theta_{0}$, we get

$$
\|\psi-f\|_{H^{1}} \leq C \epsilon(h)\|f\| .
$$

Normalize $\psi$ so that $\|\psi\|=1$, then also $\|f\|=1+o(1)$. In particular, this proves (16).

Next, $\left(P_{\theta}-z_{0}\right) \psi=\left[P_{\theta}, \chi_{B}\right] f+\chi_{B}\left(P_{\theta}-z_{0}\right) f$ and by (17), we deduce that $\left\|\left[P_{\theta}, \chi_{B}\right] f\right\| \leq C \epsilon(h)$. Therefore,

$$
\left\|\left(P_{\theta}-z_{0}\right) \psi\right\| \leq C \epsilon(h) .
$$

To pass from $P_{\theta}$ to $P$, it is enough to show that $\left\|\left(P_{\theta}-P\right) \psi\right\| \leq C \epsilon(h)$. The proof of this is based on Proposition 1. Indeed, in the coordinates considered there, 
the coefficients of $P_{\theta}-P$ are bounded by $C\left(\theta+\theta^{\prime}+\left|\theta^{\prime \prime}\right|\right)$. This, combined with Proposition 1 allows us to prove the estimate above and thus to complete the proof of the theorem.

Let $\Omega(h)$ and $\Omega_{k}(h)$ be as in Proposition 4. Apply Theorem 1 to each $\Omega_{k}$. In view of the upper bound of $b_{k}(h)-a_{k}(h)$ that we have, we see that we can choose $\epsilon(h)$ to be

$$
\epsilon(h)=C h^{-\left(3 n^{\#}+1 / 2\right)} c^{1 / 2}(h),
$$

where $C$ depends only on the constant $C\left(a_{0}, b_{0}, c_{0}\right)$ in (1). Then for each $k=$ $1, \ldots, K(h)$, Theorem 1 holds for $\Omega_{k}(h)$ with $z_{0}(h)$ there replaced by $z_{k}(h) \in$ $\left[a_{k}(h), b_{k}(h)\right]$ and $\epsilon(h)$ as above.

Definition 1 Let $\mathcal{H}^{\#}$ be a Hilbert space that can be expressed as $\mathcal{H}^{\#}=\mathcal{H}_{R_{0}} \oplus$ $L^{2}\left(B(0, R) \backslash B\left(0, R_{0}\right)\right) \oplus \mathcal{H}_{\text {ext }}, R>B+1$, with $\mathcal{H}_{\text {ext }}$ another Hilbert space, and assume that $P^{\#}(h)$ is a selfadjoint operator in $\mathcal{H}^{\#}$ with discrete spectrum in a $h$ independent neighborhood of the interval $[a(h), b(h)]$, where $a_{0} \leq a(h)<b(h) \leq b_{0}$. We call $P^{\#}(h)$ a reference operator for $P(h)$ in $\Omega(h)$ with discrepancy $\delta(h)$, if for some decomposition of $\Omega(h)$ as above such that $\operatorname{Res} P \cap \Omega=\operatorname{Res} P \cap\left(\cup_{k} \Omega_{k}\right)$ one has $\left\|\left(P^{\#}(h)-z_{k}(h)\right) \psi_{k}(h)\right\| \leq \delta(h) \rightarrow 0$, as $h \rightarrow 0$ for any $\psi_{k} \in \chi_{B} \operatorname{Ran} \Pi_{\Omega_{k}}$, $\left\|\psi_{k}\right\|=1$, where $z_{k}(h) \in \Omega_{k}(h) \cap \mathbf{R}$.

We can choose $P^{\#}(h)$ to be The Dirichlet realization of $P(h)$ restricted to a large ball, and then $\delta(h)=\epsilon(h)$. This choice of $P^{\#}(h)$ does not lead to sharp estimates however. Later we obtain $P^{\#}$ from $P$ not only by modifying it for large $x$ but also by modifying it outside the wave front set of the resonant states.

Next theorem is a "global" version of Theorem 1, i.e., it applies to resonances in wider domains $\Omega(h)$.

Theorem 2 Let $0<a_{0} \leq a(h)<b(h) \leq b_{0}, 2 e^{-h^{-1 / 3}} \leq c(h) \leq C h^{15 n^{\#}+3}$ and set

$$
\Omega(h)=[a(h), b(h)]+i[-c(h), 0] .
$$

Let $P^{\#}(h)$ be a reference operator in $\Omega(h)$ with discrepancy $\delta(h) \leq h^{9 n^{\#} / 2+1}$. Then (a)

$$
N(\Omega(h)) \leq N^{\#}\left\{\left[a(h)-\delta_{1}(h), b(h)+\delta_{1}(h)\right]\right\} \quad \text { for } h \ll 1,
$$

where $\delta_{1}(h)=h^{-9 n^{\#} / 2-1} \delta(h)$.

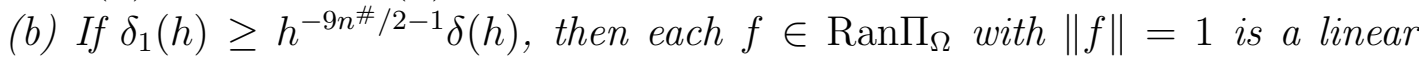
combination of eigenfunctions of $P^{\#}$ with eigenvalues in $\left[a(h)-\delta_{1}(h), b(h)+\delta_{1}(h)\right]$ up to an error that in any compact does not exceed

$$
C h^{-\left(9 n^{\#}+1\right) / 2}\left(h^{-\left(3 n^{\#}+1 / 2\right)} c^{1 / 2}(h)+\delta(h) / \delta_{1}(h)\right)
$$

for $0<h \leq h_{0}$, with $C$ and $h_{0}$ uniform with respect to the choice of $f$. 
Sketch of the proof. The basic argument in the proof is that the property that the resonant states corresponding to resonances in two different clusters in $\Omega(h)$ are linearly independent is stable under small perturbations. This property is guaranteed by Proposition 4. Let $\psi_{k}=\chi_{B} f, f_{k} \in \operatorname{Ran} \Pi_{\Omega_{k}}$ be as in Definition 1. We project $\psi_{k}$ on the space spanned by the eigenfunctions of $P^{\#}(h)$ belonging to the interval $\left.\left[a_{j}(h)-\delta_{1}(h), b_{j}(h)+\delta_{1}(h)\right]\right\} \psi_{k}(h), k=1, \ldots, K(h)$. By Theorem 1 and the spectral theorem, these projections differ from $\psi_{k}$ by $\delta(h) / \delta_{1}(h)=h^{9 n^{\#} / 2+1}$. Then we use Proposition 4, to show that the so perturbed system of functions is still linearly independent and this implies (a). Part (b) follows in a similar way.

\section{Trapped geodesics and wave front set of the resonant states}

In this section we show that in some classical situations the wave front set of the resonant states is contained in the union of the trapped rays. We consider selfadjoint differential operators of the form

$$
P(h)=\sum_{i, j=1}^{n} h D_{x_{i}} a_{i j}(x) h D_{x_{j}}+\sum_{j=1}^{n} b_{j}(x) h D_{x_{j}}+V(x)+P_{1}(h)
$$

with smooth coefficients such that $\left\{a_{i j}(x)\right\}$ is a symmetric positively definite matrix for any $x \in \mathbf{R}^{n}$ and $a_{i j}-\delta_{i j}=b_{j}=0$ for $|x|>R_{0}$ with some $R_{0}>0$ while $V(x)$ is a long range potential as above. Here $P_{1}(h)=\sum \tilde{b}_{j}(x, h) h D_{x_{j}}+\tilde{V}(x, h)$ is assumed to be a differential operator of first order with coefficients supported in $B\left(0, R_{0}\right)$, such that $P_{1}(h) \in L^{1,-1}$ considered as an $h$ - $\Psi$ DO (note that $P(h) \in L^{2,0}$ ). The operator $P(h)$ is self-adjoint in $L^{2}\left(\mathbf{R}^{n}\right)$ and satisfies the black-box assumptions.

We will use propagation of singularities results to get microlocal estimates of $\Pi_{\Omega} f /\left\|\Pi_{\Omega} f\right\|$ away from the trapping trajectories. One can define the semi-classical wave front $\mathrm{WF}^{s}(u)$ and $\mathrm{WF}(u)$ of a tempered $u$ as in [G] (see also [SjV], [I]). We note that here we will work with finite points of the semiclassical wave front set only, because we study operators with characteristic variety bounded in the $\xi$ variable. Consider the bicharacteristics of $P(h)$ related to its semi-classical principal symbol $p_{0}(x, \xi)=\sum a_{i j}(x) \xi_{i} \xi_{j}+\sum_{j} b_{j} \xi_{j}+V(x)$. They are the integral curves of the Hamiltonian vector field $H_{p_{0}}=\left(\partial_{\xi} p_{0}\right) \partial_{x}-\left(\partial_{x} p_{0}\right) \partial_{\xi}$. We call a bicharacteristic $t \mapsto \gamma(t)$ non-trapped, if for any $R>0$, there exists $\tau$ (positive or negative), such that $\gamma(\tau)$ lies outside $T^{*} B(0, R)$. We call all other bicharacteristics trapped. Denote by $\mathcal{T}$ the trapped subset of $T^{*} \mathbf{R}^{n}$, i.e, $(x, \xi) \in \mathcal{T}$ if and only if the bicharacteristic passing through $(x, \xi)$ is trapped.

Theorem 3 Let $0<a_{0} \leq a(h)<b(h) \leq b_{0}, b(h)-a(h) \leq h^{M}, M>(5 n+1) / 2$, and set

$$
\Omega(h)=[a(h), b(h)]+i\left[-h^{N}, 0\right], \quad N \geq M .
$$

Let $P(h)$ be the operator defined above and let $f=f(h) \in \operatorname{Ran}_{\Omega}$, and $\|f\|=$ 1. Then for $s=M / 2-(5 n+1) / 4, \mathrm{WF}^{s}(f)$ is supported in the set of trapped bicharacteristics of $P(h)$ on energy levels in $p_{0}^{-1}\left[a_{0}, b_{0}\right]$ uniformly with respect to the choice of $f$. More precisely, for any zeroth order symbol $q(x, \xi)$ with support disjoint 
from $\mathcal{T} \cap p_{0}^{-1}\left[a_{0}, b_{0}\right]$ there exists $C>0$ such that $\|q(x, h D) f\| \leq C h^{s}$ for any $f(h)$ as above.

If $\Omega(h)$ is as above with $0<a_{0} \leq a(h)<b(h) \leq b_{0}, N>(7 n+1) / 2$ but without smallness assumptions on $b(h)-a(h)$, then the statement of the theorem is true with $s=N / 2-(15 n+2) / 2$.

The proof of Theorem 3 is based on a propagation of singularities argument. It says that if $\left(P(h)-z_{0}(h)\right) u(h)=g(h)$ in $B(0, R),\|u(h)\|_{L^{2}(B(0, R))} \leq C$, and $u \in H^{s}$ microlocally near $\left(x_{0}, \xi_{0}\right)$, then $u \in H^{s}$ microlocally near $\left(x_{1}, \xi_{1}\right)$, provided that $\left(x_{0}, \xi_{0}\right)$ and $\left(x_{1}, \xi_{1}\right)$ can be connected by a bicharacteristic (of finite length) lying in $T^{*} B(0, R) \backslash \mathrm{WF}^{s+1}(g)$. We apply this argument to $\psi=\chi_{B} f$ that vanishes for large $x$, and therefore belongs to any $H^{s}$ there.

\section{Upper bounds on the number of resonances close to the real axis}

Let $P(h)$ be the operator (21). In this section we establish an upper bound of the resonances of $P(h)$ in a box of width independent of $h$ and height $h^{N}, N \gg 1$ in terms of the measure of the trapped set $\mathcal{T}$, where the measure is considered in $T^{*} \mathbf{R}^{n}$. To this end we choose a suitable reference operator $P^{\#}(h)$ that imposes a barrier outside a small neighborhood of the trapped set $\mathcal{T}$ by modifying $P(h)$ there. Since the resonant states are "small" there, the resonant states will be quasimodes for the new operator. An application of Theorem 2 then will imply an upper bound and

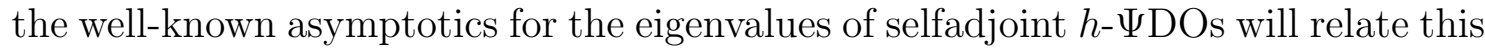
bound with meas $(\mathcal{T})$.

Theorem 4 Let $0<E_{1}<E_{2}$ be fixed and $N \geq 15 n+3$. Let $P(h)$ be as in (21) and set

$$
\Omega(h)=\left[E_{1}, E_{2}\right]+i\left[-h^{N}, 0\right] .
$$

Then

$$
N(\Omega(h)) \leq \frac{1}{(2 \pi h)^{n}}\left(\operatorname{meas}\left\{\mathcal{T} \cap p_{0}^{-1}\left[E_{1}, E_{2}\right]\right\}+o(1)\right), \quad \text { as } h \rightarrow 0,
$$

where $\mathcal{T}$ is the trapped set related to $P(h)$.

Sketch of the proof. The resonances in $\Omega(h)$ are contained in $\cup_{k=1}^{K(h)} \Omega_{k}(h)$, where $\Omega_{k}(h)$ are as in (12) with $\omega(h)$ as in Proposition 4 . Then and $b_{k}-a_{k} \leq h^{N-(7 n+1) / 2}$, $\operatorname{dist}\left\{\Omega_{k_{1}}, \Omega_{k_{2}}\right\} \geq 4 h^{N-(5 n+1) / 2}$. Each $\Omega_{k}$ satisfies the assumptions of Theorem 1. The corresponding discrepancy function (see $(20))$ is given by $\epsilon(h)=C h^{N / 2-(3 n+1 / 2)}$. Denote

$$
\mathcal{T}^{\nu}=\mathcal{T} \cap p_{0}^{-1}\left[a_{0}-\nu, b_{0}+\nu\right], \quad \mathcal{T}_{\mu}^{\nu}=\left\{\zeta \in T^{*} \mathbf{R}^{n} ; \operatorname{dist}\left\{\zeta, \mathcal{T}^{\nu}\right\}<\mu\right\},
$$

where $\nu>0, \mu>0$ are small parameters. Fix $\nu>0$. Clearly, $\mathcal{T}^{\nu}$ is a closed set. Next, $\operatorname{Vol}\left(\mathcal{T}_{\mu}^{\nu}\right) \rightarrow \operatorname{meas}\left(\mathcal{T}^{\nu}\right)$, as $\mu \rightarrow 0$. Denote $-C_{0}=\min p_{0}(x, \xi)$ and let $q_{\mu}(x, \xi)$ 
be a smooth function such that $q_{\mu}=0$ on $\mathcal{T}_{\mu}^{\nu}, q_{\mu}=2 b_{0}+C_{0}$ outside $\mathcal{T}_{2 \mu}^{\nu}$, and $0 \leq q_{\mu} \leq 2 b_{0}+C_{0}$. Set

$$
P_{\mu}^{\#}(h)=P(h)+q_{\mu}^{w}(x, h D) .
$$

Note that $P_{\mu}^{\#}(h)$ is a reference operator in $\Omega(h)$ with discrepancy $\epsilon(h)$. The principal symbol of $P_{\mu}^{\#}(h)$ is $p_{\mu}(x, \xi)=p_{0}(x, \xi)+q_{\mu}(x, \xi)$. The self-adjoint operator $P_{\mu}^{\#}(h)$ has discrete spectrum in $\left(-\infty, 2 b_{0}\right)$, because $\left\{p_{\mu}(x, \xi) \leq M\right\}$ is compact for any $M<2 b_{0}$. Moreover, we have the following estimate for the number $N_{\mu}^{\#}\left[a_{0}, b_{0}\right]$ of eigenvalues of $P_{\mu}^{\#}(h)($ see $[\mathrm{DSj}])$

$$
\frac{1}{(2 \pi h)^{n}}\left(V_{-}^{\#}\left(\left[a_{0}, b_{0}\right]\right)+o(1)\right) \leq N_{\mu}^{\#}\left[a_{0}, b_{0}\right] \leq \frac{1}{(2 \pi h)^{n}}\left(V_{+}^{\#}\left(\left[a_{0}, b_{0}\right]\right)+o(1)\right),
$$

where

$$
V_{ \pm}^{\#}\left(\left[a_{0}, b_{0}\right]\right)=\lim _{ \pm \epsilon \backslash 0} \int_{p_{\mu}(x, \xi) \in\left[a_{0}-\epsilon, b_{0}+\epsilon\right]} d x d \xi
$$

We then use the fact that $P_{\mu}^{\#}(h)$ is a reference operator to get from (23) that

$$
N(\Omega(h)) \leq \frac{1}{(2 \pi h)^{n}}\left(\operatorname{Vol}\left(p_{\mu}^{-1}\left[a_{0}-\nu, b_{0}+\nu\right]\right)+o(1)\right), \quad \text { as } h \rightarrow 0 .
$$

Next, we take the limits $\mu \rightarrow 0$ and $\nu \rightarrow 0$ to conclude the proof.

\section{Example of sharp lower bounds, generalized potential well}

In this section we study again the resonances of the operator (21) under the assumption that for some non-critical energy level $E_{2}$, the set $p_{0}^{-1}\left[-\infty, E_{2}\right]$ has at least one compact connected component. Then we get lower bound in terms of the volume of the compact component. If in addition we assume that the unbounded component is non-trapping, we also get an asymptotic formula for the resonances near the real line and a resonance free zone. This situation can be considered as a generalized "potential well in an island".

Fix two energy levels $0<E_{1}<E_{2}<\max \left\{p_{0}(x, \xi)\right\}$. Assume that $E_{1}$ and $E_{2}$ are non-critical values of $p_{0}$. Assume also that $p_{0}^{-1}\left(-\infty, E_{2}\right]$ is not connected, i.e., it has a non-empty compact component (this component then must have non-empty interior because $E_{2}$ is non-critical value of $p_{0}$ ). Then

$$
p_{0}^{-1}\left[E_{1}, E_{2}\right]=W_{\mathrm{int}} \cup W_{\mathrm{ext}},
$$

is a unbounded closed set with smooth boundary, where we denote by $W_{\text {ext }}$ the unbounded connected component and the union of the bounded ones is denoted by $W_{\text {int }}$. Then $W_{\text {int }}$ is a compact and consists of trapped points only. The set $W_{\text {ext }}$ contains non-trapped points and may contain trapped ones as well.

\section{Theorem 5}


(a) For some function $0 \leq S(h)=O\left(h^{\infty}\right)$ we have as $h \rightarrow 0$

$$
\begin{aligned}
\frac{1}{(2 \pi h)^{n}}\left(\operatorname{Vol}\left(W_{\text {int }}\right)-O(h)\right) & \leq N\left(\left[E_{1}, E_{2}\right]+i[-S(h), 0]\right) \\
& \leq N\left(\left[E_{1}, E_{2}\right]+i\left[-h^{15 n+3}, 0\right]\right) \\
& \leq \frac{1}{(2 \pi h)^{n}}\left(\operatorname{Vol}\left(W_{\text {int }}\right)+\operatorname{meas}\left\{\mathcal{T} \cap W_{\text {ext }}\right\}+o(1)\right) .
\end{aligned}
$$

(b) If $W_{\text {ext }}$ is non-trapping, i.e., if $W_{\text {ext }} \cap \mathcal{T}=\emptyset$, then there exists a function $0<S_{0}(h)=O\left(h^{\infty}\right)$ such that for any $S(h)$ such that $S_{0}(h) \leq S(h)=O\left(h^{\infty}\right)$,

$$
N\left(\left[E_{1}, E_{2}\right]+i[-S(h), 0]\right)=\frac{1}{(2 \pi h)^{n}}\left(\operatorname{Vol}\left(W_{\mathrm{int}}\right)+O(h)\right), \quad \text { as } h \rightarrow 0 .
$$

Moreover, if $P(h)=-h^{2} \Delta$ for $|x|>R_{0}$ with some $R_{0}>0$, then $\forall M>0$ the function $S(h)$ above can be chosen so that for some $h_{0}=h_{0}(M)>0$, there are no resonances in

$$
\left[E_{1}, E_{2}\right]+i[-M h,-S(h)] \text { for } 0<h<h_{0}
$$

Sketch of the proof. We show first that the $x$-projections of $W_{\text {int }}$ and $W_{\text {ext }}$ do not intersect. This allows us to use cut-off functions depending on $x$ only. Denote by $X_{\text {ext }}$ the (unique) unbounded component of $\left\{\tilde{V}(x) \leq E_{2}\right\}$ and let $X_{\text {int }}$ be the union of the connected ones. The distance between $X_{\text {int }}$ and $X_{\text {ext }}$ is positive. Let $\chi_{\text {int }}+\chi_{\text {ext }}=1$ be a partition of unity associated with those two closed sets, i.e., $\chi_{\text {int }}=1$ in a neighborhood of $X_{\text {int }}$, and $\chi_{\text {ext }}=1$ in a neighborhood of $X_{\text {ext }}$. Define

$$
\begin{aligned}
& P_{\text {int }}(h)=P(h)+V_{\text {int }}(x), \quad V_{\text {int }}(x):=\alpha \chi_{\text {ext }}(x), \\
& P_{\text {ext }}(h)=P(h)+V_{\text {ext }}(x), \quad V_{\text {ext }}(x):=\alpha \chi_{\text {int }}(x),
\end{aligned}
$$

where $\alpha>E_{2}-\inf \tilde{V}$. Then $E_{1}$ and $E_{2}$ are not critical values for neither symbol $p_{i}=$ $p_{0}(x, \xi)+V_{i}(x), i=i n t$, ext , and $p_{\text {int }}^{-1}\left[E_{1}, E_{2}\right]=W_{\text {int }}, p_{\text {ext }}^{-1}\left[E_{1}, E_{2}\right]=W_{\text {ext }}$. Moreover, $P_{\text {int }}(h)$ and $P_{\text {ext }}(h)$ are selfadjoint, $P_{\text {int }}(h)$ has discrete spectrum in $\left[E_{1}, E_{2}\right]$, while $P_{\text {ext }}(h)$ is non-trapping for energy levels in $\left[E_{1}, E_{2}\right]$.

To prove (a), note that the upper bound there follows from Theorem 4 . To prove the lower bound, use the eigenfunctions of $P_{\text {int }}(h)$ related to an $O\left(h^{\infty}\right)$ neighborhood of $\left[E_{1}, E_{2}\right]$ as quasimodes of $P(h)$. More precisely, since $P_{\text {int }}(h)$ is elliptic outside $X_{\text {int }}$, those eigenfunctions are $O\left(h^{\infty}\right)$ there (and even exponentially small there by Agmon's estimates if $P(h)$ is the Schrödinger operator), so after cutting them off near $X_{\text {int }}$, we get compactly supported asymptotically orthogonal quasimodes. An application of [St1, Theorem 1], allows us to show that there are at least as many resonances in $\Omega(h)$. This proves (a).

To prove (b), we first prove an upper bound for $N(\Omega)$ with remainder $O(h)$. Next, fix $M>0$ and assume that $z(h)$ is a resonance in the domain $\left[E_{1}, E_{2}\right]+i[-M h, 0]$. Then there exists an outgoing $u(h)$ belonging locally to the domain of $P(h)$ such that $(P(h)-z(h)) u(h)=0$. Let us normalize $u(h)$ by requiring that $\|u\|_{L^{2}(B(0, R))}=1$ with a fixed $R>R_{0}$. Notice that $P(h)-z(h)$ is elliptic for $x \notin X_{\text {int }} \cup X_{\text {ext }}$. This yields $\mathrm{WF}\left(\left.u\right|_{B\left(0, R_{0}\right)}\right) \subset T^{*}\left(X_{\text {int }} \cup X_{\text {ext }}\right)$. Choose the smooth cut-off function 
$\chi_{\text {ext }}^{\prime}$ so that $\chi_{\text {ext }}=1$ on $\operatorname{supp} \chi_{\text {ext }}^{\prime}$ and $\chi_{\text {ext }}^{\prime}=1$ in a neighborhood of $X_{\text {ext }}$. Then $(P(h)-z(h)) \chi_{\text {ext }}^{\prime} u(h)=v(h)$, where $v=\left[P(h), \chi_{\text {ext }}^{\prime}\right] u(h)=O\left(h^{\infty}\right)$ is supported in $B(0, R)$ and $\operatorname{WF}\left(\chi_{\text {ext }}^{\prime} u\right) \subset T^{*} X_{\text {ext }}$. Then $(P(h)-z(h)) \chi_{\text {ext }}^{\prime} u(h)=\left(P_{\text {ext }}(h)-\right.$ $z(h)) \chi_{\text {ext }}^{\prime} u(h)=v(h)$. Since $\chi_{\text {ext }}^{\prime} u(h)=u(h)$ for large $|x|$, we get that $\chi_{\text {ext }}^{\prime} u(h)$ is $z(h)$-outgoing. Therefore, $\chi_{\text {ext }}^{\prime} u(h)=R_{\text {ext }}(z(h), h) v(h)$, where $R_{\text {ext }}(z, h)$ is the outgoing resolvent of $P_{\text {ext }}(h)$. Since $P_{\text {ext }}$ is non-trapping for energy levels between $E_{1}$ and $E_{2}$, by [B3, Theorem 2], $\left\|\chi_{\text {ext }}^{\prime} u(h)\right\|_{L^{2}(B(0, R))} \leq(C / h)\|v(h)\|=O\left(h^{\infty}\right)$. By the ellipticity of $P(h)$ we have similar estimate for the $H^{2}$ norm of $u(h)$ near $\partial B(0, R)$ and by the trace theorem, the $H^{1}$ norm of $u(h)$ on $\partial B(0, R)$ is $O\left(h^{\infty}\right)$ as well. An application of the Green's formula in the ball $B(0, R)$ then yields $-\operatorname{Im} z=O\left(h^{\infty}\right)$. This proves part (b) of the theorem.

An example of operator satisfying the assumptions above is the Schrödinger operator $P(h)=-h^{2} \Delta+V(x)$, where $V(x)$ has strong local minimum, or more generally, if $\left\{x ; V(x) \leq E_{2}\right\}$ is not connected. In this case, the construction of the quasimodes above yields exponentially small error of the kind $e^{-d / h}$, for any $d$ is less than the Agmon distance between $X_{\text {int }}$ and $X_{\text {ext }}$. Therefore, there are resonances with exponentially small imaginary part with asymptotic number as in (a). Our proof does not exclude the existence of other resonances in the strip $\left[E_{1}, E_{2}\right]+i\left[-S(h),-e^{-d / h}\right]$ with some $S(h)=O\left(h^{\infty}\right)$ but their number does not exceed $O\left(h^{1-n}\right)$. This case has been studied in much more detail in [HSj] where other precise results are obtained.

The existence of the resonance free zone in (b) together with the results in [St3] makes it possible to get polynomial estimates on the spectral projectors $\Pi_{\Omega_{k}}$ as in Proposition 4 acting on the whole space $\mathcal{H}$ rather than on a space spanned by resonant states. This implies a resonance expansion of the solution of the corresponding wave equation as in [TZ2] and [St3]. Moreover, the wave front set of the spectral projectors are included in the trapped set and that gives us good control over the terms in that expansion.

\section{Sharp upper bounds in the classical case}

In this section we formulate a result similar to Theorem 5 in the classical case. Let $X \subset \mathbf{R}^{n}$ be a domain with smooth boundary and compact complement $\mathcal{O}$. Let

$$
P=\sum_{i, j=1}^{n} D_{x_{i}} a_{i j}(x) D_{x_{j}}+\sum_{j=1}^{n} b_{j}(x) D_{x_{j}}+V(x)
$$

be a formally symmetric elliptic differential operator with $C^{\infty}(\bar{X})$ coefficients having the same properties as those of $P(h)$ in (21). For simplicity, assume that $P=-\Delta$ for $|x|>R_{0}$. We study the resonances $\lambda$ of $P$ near the real line. Denote by $P$ again the selfadjoint realization of $P$ in $L^{2}(X)$ with Dirichlet boundary conditions on $\partial X$. We study the resonances $\lambda$ of $P$ near the real axis.

Define the generalized bicharacteristic flow of $P$ as in [MSj] (see also [H]). Recall that in the interior $T^{*} X$ the generalized bicharacteristics are the integral curves of the Hamiltonian $p_{0}(x, \xi)=\sum_{i j} a_{i j} \xi_{i} \xi_{j}$. We assume that the bicharacteristics of $P$ cannot be tangent to the boundary of infinite order. Under this assumption, any 
generalized bicharacteristics is uniquely determined by any of its points. Define the trapped subset $\mathcal{T}$ of $T^{*} X$ as the complement of the set of all $\zeta \in T^{*} X$, for which any generalized bicharacteristic passing through $\zeta$ leaves $B\left(0, R_{0}\right) \times \mathbf{R}^{n}$ for either $t>0$ or $t<0$. Fix a decreasing function $0<S(r)=O\left(r^{-\infty}\right)$, as $r \rightarrow \infty$. Set

$$
\Omega(r):=\{\lambda \in \mathbf{C} ; 1 \leq \operatorname{Re} \lambda \leq r, 0<-\operatorname{Im} \lambda<S(\operatorname{Re} \lambda)\} .
$$

The main result in this section is the following.

Theorem 6 Let $P$ be the operator (26) and $\Omega(r)$ be as in (27). Then

$$
N(\Omega(r)) \leq \frac{r^{n}}{(2 \pi)^{n}}\left(\operatorname{meas}\left(\mathcal{T} \cap B^{*} X\right)+o(1)\right), \quad \text { as } r \rightarrow \infty,
$$

where $B^{*} X=\left\{(x, \xi) \in T^{*} X ; p_{0}(x, \xi) \leq 1\right\}$.

To prove the theorem, we translate the problem into a semiclassical one. We use the propagation of singularities results in [MSj]. We construct a reference operator $P^{\#}(h)$ by imposing a pseudodifferential barrier near $\mathcal{T}$ as above but we do that outside a small neighborhood of the boundary only. Near the boundary, our operator remains the same. Then we extend the operator in the whole space. The cut-off resonant states, extended as zero inside the obstacle, do not belong locally to $H^{2}$ and are not in the domain of the reference operator $P^{\#}(h)$. We work with the quadratic form $\left(P^{\#}(h) f, f\right)_{L^{2}\left(\mathbf{R}^{n}\right)}$ however, and they do belong to its domain. Using the min-max principle, we prove the desired estimate.

We would like to give an example of a system with trapped set of positive measure. Let $P=-\Delta$ in the exterior of a bounded obstacle with smooth boundary and assume that there exists an elliptic periodic ray satisfying some mild degeneracy conditions (see [Po]). Then it is known that for some $S(r)=O\left(r^{-\infty}\right), N(\Omega(r)$ ) admits a lower bound of the kind $c r^{n}(1+o(1))$. The constant $c$ there is positive and is proportional to the measure of the invariant tori around the elliptic ray, which existence is guaranteed by the KAM theory. This constant can also be chosen to be $c=(2 \pi)^{-n}$ meas $\left(\mathcal{T}_{0} \cap B^{*} X\right)$, where $\mathcal{T}_{0} \subset \mathcal{T}$ is a Cantor set of trapped rays near the periodic elliptic ray. There is no hope that meas $\left(\mathcal{T}_{0} \cap B^{*} X\right)=\operatorname{meas}\left(\mathcal{T} \cap B^{*} X\right)$ because $\mathcal{T}_{0}$ is (a part of) the trapped rays that are close enough to a single periodic ray, while $\mathcal{T}$ is the set of all trapped rays. Nevertheless, this gives us a two-side estimate with different constants in the principal terms that have the same nature.

\section{References}

[B2] N. BURQ, Lower bounds for shape resonances widths of long range Schrödinger operators, preprint.

[B3] N. BurQ, Semi-classical estimates for the resolvent in non-trappimg geometries, preprint.

[G] C. GÉRARD, Asymptotique des poles de la matrice de scattering pour deux obstacles strictement convex, Bull. Soc. Math. France, Mémoire n. 31, 116, 1988. 
[DSj] M. Dimassi And J. SuÖstrand, Spectral Asymptotics in the Semi-Classical Limit, London Math. Society Lecture Notes Series, No. 268, Cambridge Univ. Press, 1999.

[HSj] B. Helffer, J. Sjöstrand, Résonances en limite semi-classique, Mém. Soc. Math. France (N.S.), 24-25, 1986.

[H] L. Hörmander, The Analysis of Linear Partial Differential Operators, Springer-Verlag, Berlin, 1985.

[I] V. IVRII, Microlocal Analysis and Precise Spectral Asymptotics, SpringerVerlag, Berlin, 1998.

[K] T. Kato, Perturbation Theory for Linear Operators. Springer-Verlag, New York, 1966.

[LZ] K. LIN AND M. ZWORSKI, Resonances in chaotic scattering, www.math.berkeley.edu/ kkylin/resonances/.

[MSj] R. Melrose and J. Sjöstrand, Singularities of boundary value problems. I, II. Comm. Pure Appl. Math. 31(1978), no. 5, 593-617, and 35(1982), no. 2, $129-168$.

[Po] G. Popov, Quasi-modes for the Laplace operator and Glancing hypersurfaces, in: Proceedings of Conference on Microlocal analysis and Nonlinear Waves, Minnesota 1989, M. Beals, R. Melrose and J. Rauch eds., Springer Verlag, Berlin-Heidelberg-New York, 1991.

[Sj1] J. SJÖSTRAND, Geometric bounds on the density of resonances for semiclassical problems, Duke Math. J. 60(1)(1990), 1-57.

[Sj2] J. Sjöstrand, A trace formula and review of some estimates for resonances, in Microlocal analysis and Spectral Theory (Lucca, 1996), 377-437, NATO Adv. Sci. Inst. Ser. C Math. Phys. Sci., 490, Kluwer Acad. Publ., Dordrecht, 1997.

[SjV] J. Sjöstrand and G. Vodev, Asymptotics of the number of Rayleigh resonances, Math. Ann. 309(1997), 287-306.

[SjZ] J. SuÖSTRAND AND M. ZwOrski, Complex scaling and the distribution of scattering poles, Journal of AMS 4(4)(1991), 729-769.

[St1] P. Stefanov, Quasimodes and resonances: sharp lower bounds, Duke Math. J. 99(1999), 75-92.

[St2] P. Stefanov, Lower bound of the number of the Rayleigh resonances for arbitrary body, Indiana Univ. Math. J. 49(2)(2000), 405-426.

[St3] P. Stefanov, Resonance expansions and Rayleigh waves, Math. Res. Lett., 8(1-2)(2001), 105-124. 
[StV1] P. Stefanov And G. Vodev, Distribution of resonances for the Neumann problem in linear elasticity outside a strictly convex body, Duke Math. J. 78(1995), 677-714.

[TZ1] S.-H. TANG AND M. Zworski, From quasimodes to resonances, Math. Res. Lett., 5(1998), 261-272.

[TZ2] S.-H. TANG AND M. ZwOrski, Resonance expansions of scattered waves, Comm. Pure Appl. Math. 53(10)(2000), 1305-1334.

[V] G. Vodev, Sharp polynomial bounds on the number of scattering poles for perturbations of the Laplacian, Comm. Math. Phys. 146(1992), 39-49.

[Ze] M. ZERZERI, Majoration du nombre des résonances près de l'axe reél pour une perturbation, à support compacte, abstraite, du laplacien, perpint, 20002001, Univ. Paris 13.

[Z1] M. Zworski, Sharp polynomial bounds on the number of scattering poles, Duke Math. J. 59(1989), 311-323.

[Z2] M. ZwORSKI, private communication, 1992.

Department of Mathematics, Purdue University, WeSt LAFAYETTE, IN 47907, USA

stef anov@math . purdue . edu

www.math.purdue.edu/ stefanov 